\title{
A SHARP LOWER BOUND FOR THE SCALAR CURVATURE OF CERTAIN STEADY GRADIENT RICCI SOLITONS
}

\author{
MANUEL FERNÁNDEZ-LÓPEZ AND EDUARDO GARCÍA-RÍO
}

(Communicated by Lei Ni)

\begin{abstract}
We give a sharp lower bound for the scalar curvature of a steady gradient Ricci soliton in terms of the hyperbolic secant of the distance from a fixed point under the assumption that $2|R c|^{2} \leq R^{2}$, a condition that is satisfied by any steady gradient Ricci soliton with nonnegative sectional curvature.
\end{abstract}

A steady Ricci soliton is a Riemannian manifold $(M, g)$ that admits a smooth vector field $X$ on $M$ such that

$$
\frac{1}{2} \mathcal{L}_{X} g+R c=0
$$

where $\mathcal{L}_{X}$ is the Lie derivative in the direction of the vector field $X$ and $R c$ denotes the Ricci tensor. When the vector field $X$ may be replaced by the gradient of some smooth function $f$ on $M$, called the potential function, $(M, g)$ is said to be a steady gradient Ricci soliton. In such a case the Ricci soliton equation becomes

$$
R c+H_{f}=0,
$$

where $H_{f}$ denotes the Hessian of the function $f$.

It is well-known (see 1, 2, for example) that the scalar curvature and the norm of the potential function of a steady gradient Ricci soliton are related by $R+|\nabla f|^{2}=$ $C$, where $C$ is a positive constant, unless the steady soliton is Ricci flat and $f$ is constant. Moreover, the metric can be scaled so that $R+|\nabla f|^{2}=1$, and we assume it in what follows.

Lower bounds for the scalar curvature of steady gradient Ricci solitons were investigated by Chow, Lu and Yang [4], who showed that $R \geq\left(2+\sqrt{\frac{n}{2}}\right)^{-1} e^{f}$ provided that the potential function is nonpositive and $\lim _{x \rightarrow \infty} f(x)=-\infty$. Since the scalar curvature of a steady gradient Ricci soliton is $R \geq 0,|\nabla f|^{2} \leq 1$ and one has that $f(x) \geq f(O)-r(x)$, where $O$ is a fixed point on $M$ and $r(x)$ denotes the radial distance $r(x)=d(x, O)$. Thus the lower bound obtained in 4 can be expressed in terms of the distance function from a fixed point, showing that the scalar curvature decays at most exponentially in the distance.

The main result of this note is the following:

Theorem 1. Let $(M, g)$ be a complete gradient steady Ricci soliton normalized so that $R+|\nabla f|^{2}=1$. If $-R / 2 \leq R c \leq R / 2$, then

$$
R(x) \geq k \operatorname{sech}^{2} \frac{r(x)}{2},
$$

Received by the editors October 6, 2011.

2010 Mathematics Subject Classification. Primary 53C25, 53C20, 53C44.

Key words and phrases. Gradient Ricci soliton, scalar curvature.

(c) 2013 American Mathematical Society 2145 
where $r(x)$ is the distance from a fixed point $O \in M$ and $k \leq 1$ is a constant that only depends on the point $O \in M$ and the value $R(O)$.

Remark 2. On the scaled Hamilton's cigar soliton $\left(\mathbb{R}^{2}, \frac{4}{1+x^{2}+y^{2}}\left(d x^{2}+d y^{2}\right)\right)$ one has $R+|\nabla f|^{2}=1$ and $R(x)=\operatorname{sech}^{2} \frac{r(x)}{2}$, where the distance $r(x)$ is measured from the only point where the scalar curvature attains its maximum. This shows that the lower bound in Theorem 1 is sharp in dimension two.

In higher dimensions, the product of the Hamilton's cigar soliton and any complete Ricci flat manifold shows that (0.1) is sharp. Indeed, note that we actually have equality when moving in the direction of the cigar, where the distance $r(x)$ is measured from one of the points where the scalar curvature attains its maximum.

Note that the previous result applies to steady gradient Ricci solitons with nonnegative sectional curvature.

Lemma 3. Let $(M, g)$ be a Riemannian manifold with nonnegative curvature. Then $2|R c|^{2} \leq R^{2}$.

Proof. Let $\left\{E_{1}, E_{2}, \ldots, E_{n}\right\}$ be a local orthonormal frame consisting of eigenvectors of the Ricci tensor. Then

$$
\begin{aligned}
-R_{i i}+\frac{1}{2} R & =\frac{1}{2}\left(\sum_{j \neq i} R_{j j}-R_{i i}\right) \\
& =\frac{1}{2} \sum_{j \neq i}\left(R_{j j}-R_{j i i j}\right) \\
& =\frac{1}{2} \sum_{j \neq i, k \neq i, k \neq j} R_{k j j k} \geq 0 .
\end{aligned}
$$

Now $0 \leq \frac{1}{2} R g_{i j}-R_{i j}$ and $0 \leq R_{i j}$, from which it follows that $0 \leq\left(\frac{1}{2} R g_{i j}-R_{i j}\right) R_{i j}$ $=\frac{1}{2} R^{2}-|R c|^{2}$.

A lower bound for the scalar curvature of steady gradient Ricci solitons with nonnegative Ricci tensor is obtained as follows.

Theorem 4. Let $(M, g)$ be a complete steady gradient Ricci soliton with nonnegative Ricci curvature and normalized so that $R+|\nabla f|^{2}=1$. Then

$$
R(x) \geq k \operatorname{sech}^{2} r(x),
$$

where $r(x)$ is the distance from a fixed point $O \in M$ and $k \leq 1$ is a constant that only depends on the point $O \in M$ and the value $R(O)$.

Remark 5. Deciding whether (0.2) is sharp is an open question. Indeed, we do not know of any example where the equality is achieved.

Note that the infimum of the scalar curvature (and of the norm of the Ricci tensor) on a steady gradient Ricci soliton is 0 , as shown in [5] by using maximum principles. Since $\Delta_{f} R=-2|R c|^{2}$, one has that $R$ does not attain a local minimum unless it is constant. So we have the following:

Theorem 6. Let $(M, g)$ be a complete steady gradient Ricci soliton. Then

$$
\liminf _{d(x, O) \rightarrow \infty} R(x)=\liminf _{d(x, O) \rightarrow \infty}|R c(x)|^{2}=0,
$$

where $O$ is any fixed point on $M$. 
Remark 7 . The previous result shows that the scalar curvature of any complete steady gradient Ricci soliton decays to zero at infinity, at least in some direction. Related to this result, it is shown in [8] that $\liminf _{d(x, O) \rightarrow \infty} R(x)=0$, studying the behaviour of the potential function, and in 3 . the authors showed that $\liminf _{d(x, O) \rightarrow \infty}|R c(x)|^{2}=0$ by a different way.

Proof of Theorem 1. From Kato's inequality, at every point where $\nabla f$ does not vanish, we have

$$
\left|H_{f}\right|^{2}=|\nabla \nabla f|^{2} \geq\left.|\nabla| \nabla f\right|^{2}=|\nabla \sqrt{1-R}|^{2}=\frac{|\nabla R|^{2}}{4|\nabla f|^{2}}
$$

or, equivalently,

$$
|\nabla R|^{2} \leq 4\left|H_{f}\right|^{2}|\nabla f|^{2}
$$

By smoothness, the previous inequality is satisfied on all $M$. Now, under our assumption we have that $\left|H_{f}\right|^{2}=|R c|^{2} \leq \frac{R^{2}}{2}$. Thus

$$
\frac{|\nabla R|}{R \sqrt{1-R}} \leq 1
$$

Next, let $O$ be a fixed point on $M$ and let $\gamma:[0, t] \rightarrow M$ be a minimizing geodesic with $\gamma(0)=O$. Integrating the function $\frac{-(R \circ \gamma)^{\prime}}{R \sqrt{1-R}}$ along $\gamma(s)$ we get

$$
\left[\ln \frac{1+\sqrt{1-R}}{1-\sqrt{1-R}}\right]_{0}^{t}=-\int_{0}^{t} \frac{(R \circ \gamma)^{\prime}}{R \sqrt{1-R}} d s \leq \int_{0}^{t} \frac{|\nabla R|}{R \sqrt{1-R}} d s \leq t .
$$

Put $c=\frac{1+\sqrt{1-R(O)}}{1-\sqrt{1-R(O)}}$ to get

$$
1+\sqrt{1-R(\gamma(t))} \leq c e^{t}(1-\sqrt{1-R(\gamma(t))}) .
$$

Now a straightforward computation shows that

$$
R(\gamma(t)) \geq \frac{4 c}{c^{2} e^{t}+2 c+e^{-t}}
$$

and, since $c \geq 1$, we have that

$$
R(\gamma(t)) \geq \frac{4 c}{c^{2} e^{t}+2 c+e^{-t}} \geq \frac{4 c}{c^{2} e^{t}+2 c^{2}+c^{2} e^{-t}}=\frac{1}{c} \operatorname{sech}^{2} \frac{t}{2},
$$

which finishes the proof.

Remark 8 . Note that the scalar curvature $R$ may take the value 1 along the geodesic $\gamma([0, t])$. Since gradient Ricci solitons are analytic manifolds (see [6]) we have two possibilities. If the set of points where $R$ takes the value 1 on $\gamma([0, t])$ has a point of accumulation in $\gamma([0, t])$, then $R$ must be constant, because of analyticity, and our claim is trivially true. In the other case, we have only a finite set of points $\gamma([0, t])$ where $R$ takes the value 1 . Then we have to deal, eventually, with a finite number of improper integrals, and the proof works.

Remark 9. Note that the inequality $|\nabla R|^{2} \leq 4\left|H_{f}\right|^{2}|\nabla f|^{2}$ in the previous proof also follows from the fact that for any gradient Ricci soliton $\nabla R=2 R c(\nabla f)$, and thus in the steady case one has $|\nabla R|^{2} \leq 4|R c|^{2}|\nabla f|^{2}=4\left|H_{f}\right|^{2}|\nabla f|^{2}$, without using Kato's inequality. 
Proof of Theorem 4. Proceeding as in the proof of Theorem 1 and using the inequality $\left|H_{f}\right|^{2}=|R c|^{2} \leq R^{2}$ we get $\frac{|\nabla R|}{R \sqrt{1-R}} \leq 2$. Then the result is obtained as in the above.

\section{ACKNOWLEDGMENTS}

The authors were supported by projects MTM2006-01432 and PGIDIT06PXIB 207054PR (Spain).

\section{REFERENCES}

[1] H.-D. Cao; Recent progress on Ricci solitons, Recent advances in geometric analysis, 1-38, Adv. Lect. Math. (ALM), 11, Int. Press, Somerville, MA, 2010. MR2648937 (2011d:53061)

[2] B. Chow, S.-C. Chu, D. Glickenstein, Ch. Guenther, J. Isenberg, T. Ivey, D. Knopf, P. Lu, F. Luo and L. Ni; The Ricci flow: Techniques and applications. Part I. Geometric aspects. Mathematical Surveys and Monographs, 135, American Mathematical Society, Providence, RI, 2007. MR2302600 (2008f:53088)

[3] B. Chow and P. Lu; Mild Ricci curvature restrictions for steady gradient Ricci solitons, arXiv:1103.5505v2.

[4] B. Chow, P. Lu and B. Yang; Lower bounds for the scalar curvatures of noncompact gradient Ricci solitons, C. R. Math. Acad. Sci. Paris 349 (2011), 1265-1267. MR2861997

[5] M. Fernández-López and E. García-Río; Maximum principles and gradient Ricci solitons, J. Differential Equations 251 (2011), 73-81. MR2793264 (2012d:53136)

[6] T. A. Ivey; Local existence of Ricci soltions, Manuscripta Math. 91 (1996), 151-162. MR.1411650 (98e:53063)

[7] P. Petersen and W. Wylie; Rigidity of gradient Ricci solitons, Pacific J. Math. 241 (2009), 329-345. MR2507581 (2010j:53071)

[8] P. Wu; On the potential function of gradient steady Ricci solitons, J. Geom. Anal., to appear. DOI:10.1007/s12220-011-9243-7

Faculty of Mathematics, University of Santiago de Compostela, 15782 Santiago de Compostela, Spain

E-mail address: manuf1@edu.xunta.es

Faculty of Mathematics, University of Santiago de Compostela, 15782 Santiago de Compostela, Spain

E-mail address: eduardo.garcia.rio@usc.es 